\title{
Особенности дефектообразования в наноструктурированном кремнии при ионном облучении
}

\author{
(C) А.В. Кожемяко ${ }^{1}$, А.П. Евсеев ${ }^{1,2}$, Ю.В. Балакшин ${ }^{2}$, А.А. Шемухин ${ }^{2}$ \\ ${ }^{1}$ Московский государственный университет им. М.В. Ломоносова (физический фракультет), \\ 119991 Москва, Россия \\ ${ }^{2}$ Научно-исследовательский институт ядерной физики им. Д.В. Скобельцына, \\ 119991 Москва, Россия \\ E-mail: anastasiia.kozhemyako@mail.ru
}

Поступила в Редакцию 18 декабря 2018 г.

В окончательной редакции 25 декабря 2018 г.

Принята к публикации 25 декабря 2018 г.

\begin{abstract}
Проведено облучение наноструктурированного кремния ионами $\mathrm{Si}^{+}$и $\mathrm{He}^{+}$с энергиями 200 и 150 кэВ соответственно. Методом комбинационного рассеяния света исследовано разрушение структуры облученных образцов, накопление дефектов при различных дозах облучения. Показано, что пленки монокристаллического кремния аморфизуются под действием облучения при величине смещений на атом 0.7. Однако пористый кремний при величине смещений на атом 0.5 не аморфизуется полностью, в спектрах комбинационного рассеяния наблюдается слабый сигнал, соответствующий аморфной фазе кремния, и в то же время присутствует явный сигнал от кристаллической фазы кремния. Проведена оценка размеров нанокристаллитов в структуре пористого кремния при разных дозах облучения.
\end{abstract}

DOI: 10.21883/FTP.2019.06.47734.9050

\section{1. Введение}

Существует несколько модификаций наноструктурированного кремния: нанонити [1], пористые структуры [2-4], пленки кремния, полученные при газофазном осаждении [5]. Мезо- и нанокремний находит свое применение в ряде приложений электроники, фотоники и тераностики. Он является отличным материалом для изготовления оптических устройств, таких как волноводы, фотонные кристаллы, оптические фильтры и т.д. [6]. Оптические свойства $[7,8]$ пористого кремния были успешно использованы для изготовления датчиков и биосенсоров: например, мезопористый кремний использовался в качестве многоразового биокаталитического устройства [9]. Мезо- и наночастицы кремния биоразлагаемы и биосовместимы $[10]$, являются отличными кандидатами для биосенсоров in vivo [11] и позволяют проводить in vitro диагностику раковых заболеваний на ранних стадиях [12]. Частицы пористого кремния субмикрометрового размера (500-600 нм) являются многообещающим средством для дисперсных транспортных систем направленной лекарственной доставки [13].

В связи с тенденцией использования наночастиц кремния в наноэлектронике и биомедицинских направлениях возникает необходимость развития методик, позволяющих как контролировать, так и модифицировать его физические свойства с высокой точностью и повторяемостью результата воздействия, например, путем инженерии дефектов. Воздействие пучками ионов является перспективным методом модификации состава и структуры вещества. Оно позволяет точно контролировать концентрации и распределения внедряемых элементов. Дефекты, образующиеся при ионном облучении, из- меняют электронные свойства материалов, которые в свою очередь влияют на такие свойства, как поглощение света, люминесценция, перенос и разделение носителей заряда $[14,15]$.

Уже сегодня ионная имплантация активно используется для модификации свойств наноструктур, что подтверждается большим числом фундаментальных и прикладных работ. Например, облучение ионами аргона кремниевых структур привело к сдвигу максимума пика в спектрах отражения падающего света в область меньших длин волн по сравнению с обычным пористым кремнием [16]. Облучение пленок $\mathrm{SiO}_{2}$ ионами $\mathrm{Si}^{+}$и $\mathrm{C}^{+}$с различными дозами позволяет эффективно управлять длиной волны фотолюминесценции [17]. При облучении монокристалла германия ионами кремния германий переходит из фазы $c$-Ge в фазу $a$-Ge, а на его поверхности образуются поры [18]. Проявление наноразмерных эффектов может привести к резким изменениям электрических, оптических свойств $[19,20]$ по сравнению с массивными материалами.

Кроме модификации состава и структуры пучки ионов используют для модификации топографии поверхности. Например, с помощью облучения поверхности монокристалла кремния и слоев нитрида кремния ионами кислорода и аргона $[21,22]$ можно варьировать угол смачиваемости, а облучение пучком кластерных ионов аргона [23] кремниевых фотонных структур приводит к сглаживанию поверхности пористого кремния и последующему усилению отражения света.

Таким образом, ионное облучение уже сейчас активно используется для улучшения характеристик приборов, созданных на основе наноструктур. Однако параметры ионного облучения для наноразмерных материалов значительно отличаются от параметров для массивных 
структур ввиду наличия развитой поверхности и резкой границы раздела. Например, в предыдущих работах авторов [24,25] было показано, что критическая доза аморфизации для пленок кремния значительно отличается от таковой для массивных образов. Однако для диагностики кристалличности использовался метод резерфордовского обратного рассеяния, что может вносить дополнительные трудности (время, вакуум и деградация образца) при анализе наноразмерных структур типа пористого кремния.

Поэтому в данной работе модификация структуры пленки монокристаллического кремния на сапфире и пористого кремния под воздействием облучения ионами $\mathrm{He}^{+}$и $\mathrm{Si}^{+}$изучена с помощью комбинационного рассеяния света и проведено сравнение процессов дефектообразования в пленках и массивном кремнии, а также в пористых структурах.

\section{2. Экспериментальные методы}

Пористый кремний получен методом анодной поляризации в специальных электрохимических ячейках $[26,27]$. Структура пористого слоя определяется плотностью тока, концентрацией HF в электролите и стехиометрией образца [28]. В данной работе в качестве анода использовали кремниевую пластину Si (100), которая помещалась в ячейку из фторопласта. Травление проводили в раствоpe $\mathrm{HF}(49 \%): \mathrm{C}_{2} \mathrm{H}_{5} \mathrm{OH}$ в соотношении $1: 1$ при плотности тока $60 \mathrm{MA} / \mathrm{cm}^{2}$. Толщина пленки пористого кремния практически линейно зависит от времени травления и изменяется в пределах от долей до сотен микрометров. Глубина травления на изображениях, полученных методом сканирующей электронной микроскопии (СЭМ), сколов образцов превышала 3 мкм. Для дальнейшего исследования отбирались участки кремниевой пластины, удаленные от края области травления, чтобы избежать эффектов от неоднородности.

Кроме пористого кремния в работе использовались пленки монокристаллического кремния на сапфире с ориентацией (100), толщина пленки составляла 300 нм. Образцы представляли собой квадраты размером $1 \times 1 \mathrm{~cm}$.

Облучение проводилось на ускорителе HVEЕ-500. Линия имплантации содержит системы фокусировки ионного пучка и сканирования мишени, а также ловушку нейтральных частиц. Рабочее давление в камере не превышало $10^{-5}$ Па.

Для удобства сравнения процессов накопления дефектов дозы облучения представлены не только в $\mathrm{cm}^{-2}$, но и в единицах dpa. Единицы измерения дозы dpa (displacements per atom, число смещений на атом) являются универсальными. Использование этого подхода позволяет сравнивать процессы дефектообразования, вызванные ионным облучением, при различных энергиях,

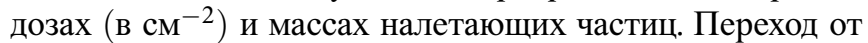
доз в $\mathrm{cm}^{-2}$ к dра осуществлялся в соответствии с [29].

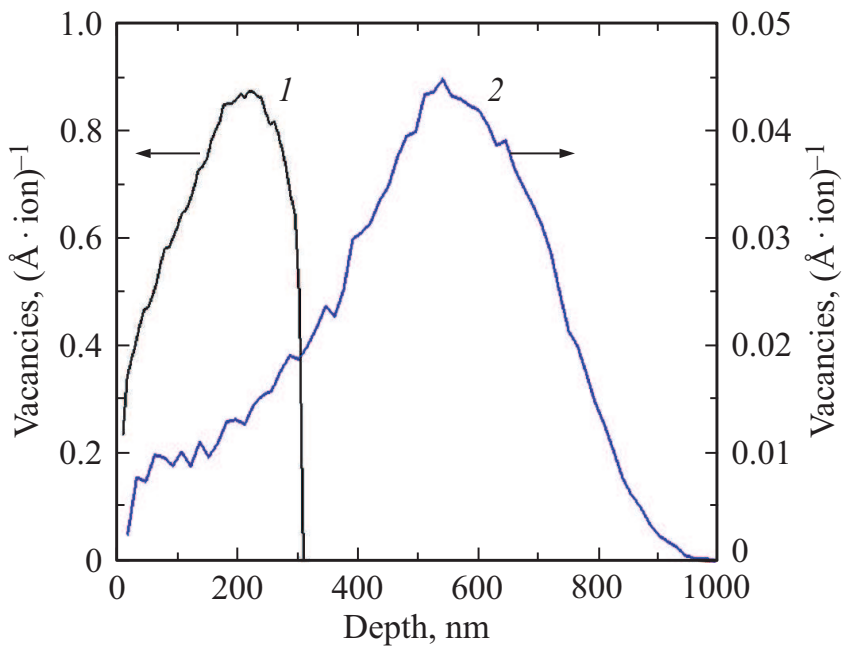

Рис. 1. Профиль распределения вакансий, образовавшихся в монокристаллическом кремнии после облучения ионами кремния с энергией 200 кэВ (1) и образовавшихся в пористом кремнии после облучения ионами гелия с энергией 150 кэВ (2).

Для каждой использованной дозы значение dра было получено как отношение полной концентрации вакансий, образовавшихся при облучении ионами на глубине максимума упругих потерь, к атомной концентрации кремния. Для кристаллического кремния атомная концентрация $n_{c-\mathrm{Si}}=4.97 \cdot 10^{22} \mathrm{~cm}^{-3}$. Пористость кремния после травления измерялась гравиметрическим методом в соответствии с [30] и составила $0.5 n_{c-\mathrm{Si}}$. В моделировании дефектообразования в пористом кремнии применялось следующее приближение. Поскольку структура пористого кремния por-Si представляет собой монокристаллический остов, содержащий полости, расчет профиля распределения вакансий в SRIM проводился при плотности вещества $n_{c-\mathrm{Si}}$, а вероятность взаимодействия налетающего иона с атомами мишени уменьшилась в 2 раза.

Профили распределения индуцированных облучением вакансий были рассчитаны при помощи программы SRIM [31,32]. Результаты моделирования приведены на рис. 1.

Известно, что доза аморфизации для массивных полупроводников > $10 \mathrm{dpa}$ [33]. При этом пленки кремния аморфизуются при меньших дозах [34].

Параметры проведенных экспериментов и значения доз облучения в единицах dpa приведены в таблице.

Параметры экспериментов

\begin{tabular}{c|c|c|c}
\hline \multirow{2}{*}{ Подложка } & \multirow{2}{*}{ Тип } & \multicolumn{2}{|c}{ Доза } \\
\cline { 3 - 4 } & и энергия иона & DPA & $\mathrm{cm}^{-2}$ \\
\hline \multirow{2}{*}{$c-\mathrm{Si}$} & \multirow{2}{*}{$\mathrm{Si}^{+}, 200$ кэB } & 0.7 & $4 \cdot 10^{14}$ \\
& & 1.2 & $7 \cdot 10^{14}$ \\
& & 1.7 & $10^{15}$ \\
\hline \multirow{2}{*}{ por-Si } & \multirow{2}{*}{$\mathrm{He}^{+}, 150$ кэB } & 0.05 & $10^{15}$ \\
& & 0.5 & $10^{16}$
\end{tabular}


Чаще всего для анализа дефектообразования при ионном облучении используют ширину качания рентгеновского луча [35-36], оптические методы [37] и резерфордовское обратное рассеяние [38]. В данной работе анализ степени разупорядочения был проведен с помощью комбинационного рассеяния света. Спектроскопия комбинационного рассеяния является одним из основных методов исследования структурных особенностей материалов, позволяющих исследовать дефекты и степень аморфизации структуры [39-41]. Спектры комбинационного рассеяния (КР) были получены при комнатной температуре на установке „Зондовая нанолаборатория ИНТЕГРА Спектра“ с использованием лазера с длиной волны излучения 632.8 нм, мощность лазера при измерении составляла $7 \mathrm{MBT} / \mathrm{cm}^{2}$.

\section{3. Результаты и обсуждение}

На рис. 2 представлены спектры комбинационного рассеяния света, полученные от монокристаллического кремния, облученного ионами $\mathrm{Si}^{+}$с энергией 200 кэВ и дозами от $4 \cdot 10^{14}$ до $10^{15} \mathrm{~cm}^{-2}$. В спектрах образцов, полученных при двух меньших дозах облучения, виден отчетливый пик при $520 \mathrm{~cm}^{-1}$, соответствующий кристаллической фазе кремния. Присутствующий во всех спектрах широкий пик с максимум при $480 \mathrm{~cm}^{-1}$ соответствует аморфной фазе кремния. В работе [42] пики при 480 и $520 \mathrm{~cm}^{-1}$ также относятся к аморфной и кристаллической фазам соответственно. Снижение интенсивности пика при 520 и рост интенсивности пика при $480 \mathrm{~cm}^{-1}$ с ростом дозы облучения говорит о разупорядочении кристаллической структуры образца. Незначительная интенсивность пика $520 \mathrm{~cm}^{-1}$ в спектре, соответствующем дозе $10^{15} \mathrm{~cm}^{-2}$, говорит о практически полной аморфизации модифицированного облучением слоя, что подтверждается результатами измерения спектров резерфордовского обратного рассеяния (РОР) [25].

Приведенные спектры не содержат пиков, характерных для комбинационного рассеяния света на сапфире в указанном диапазоне: $378,418,432,415,578$ и $645 \mathrm{~cm}^{-1}$ [43]. Таким образом, можно сделать вывод, что глубина анализа спектроскопии комбинационного рассеяния не превышает 300 нм для монокристаллического кремния.

В работе [44] в результате исследования облученного кремния методом электронного парамагнитного резонанса (ЭПР) авторы предположили, что при определенной дозе облучения в монокристаллическом кремнии происходит образование нанокристаллов. Монокристалл кремния облучался ионами $\mathrm{Ge}^{+}, \mathrm{Ar}^{+}$и $\mathrm{He}^{+}$с энергиями от 80 до 150 кэВ и дозами от $2 \cdot 10^{13}$ до $5 \cdot 10^{16} \mathrm{~cm}^{-2}$, что при переходе к единицам dра составило от 0.1 до 56.0 dра. Было обнаружено, что зависимость числа парамагнитных центров, определенная методом ЭПР, от дозы содержит максимум. Данный характер зависимости повторяется для всех рассмотренных типов ионов.

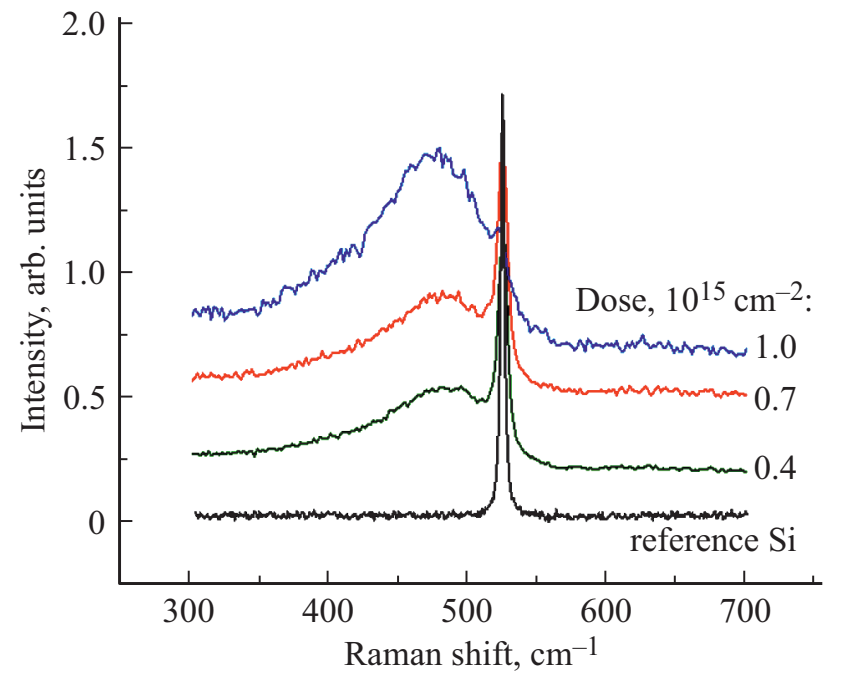

Рис. 2. Интенсивность спектра комбинационного рассеяния света, полученного от пленок кремния после облучения ионами Si с энергией 200 кэВ.

Авторы работы объясняют возникновение данного максимума образованием наночастиц кремния, окруженных аморфной фазой.

Также необходимо отметить, что, согласно спектрам, представленным на рис. 2, смещения пика $520 \mathrm{~cm}^{-1}$ не происходит. В работах $[40,42]$ также не наблюдается значительного сдвига пика, соответствующего кристаллической фазе кремния.

Далее было проведено исследование дефектообразования в пористом кремнии. Исследуемые образцы изначально представляли собой пластины кремния диаметром 10 см, на которых был сформирован слой пористого кремния толщиной $>3$ мкм. Затем из однородного участка в центре пластины нарезали образцы размером $1 \times 1 \mathrm{~cm}$, которые затем облучались в имплантационной камере.

На рис. 3 приведены спектры КР пористого кремния после облучения ионами $\mathrm{He}^{+}$с энергией 150 кэВ, дозами $10^{15}$ и $10^{16} \mathrm{~cm}^{-2}$. Облучение проводилось под углом $45^{\circ}$ к нормали для увеличения количества столкновений ионов с атомами мишени. В спектре от необлученной структуры присутствует узкий пик на частоте $520 \mathrm{~cm}^{-1}$, соответствующий кристаллической фазе кремния. С poстом дозы облучения снижается интенсивность данного пика и происходит смещение максимума в область меньших волновых векторов.

Смещение пика, соответствующего сигналу от кристаллической фазы кремния, может говорить о присутствии в структуре нанокристаллов, что приводит к ослаблению правил отбора по $k$. Увеличение смещения может объясняться разрушением нанокристаллов. Смещение пика при $520 \mathrm{~cm}^{-1}$ в работе [42] также объясняется наличием и изменением размера нанокристаллов. Уширение пика может быть вызвано накоплением дефектов в приповерхностном слое образца, аналогичное 


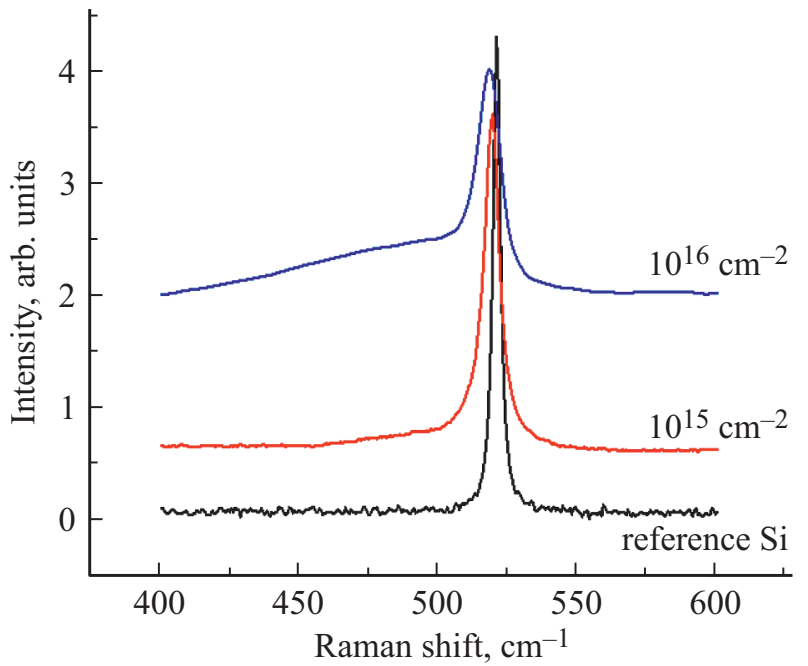

Рис. 3. Спектры комбинационного рассеяния света, полученного от пленок пористого кремния после облучения ионами $\mathrm{He}^{+}$с энергией 150 кэВ, дозами $10^{15}, 10^{16} \mathrm{~cm}^{-2}$.

объяснение дано в работе [40]. Падение интенсивности объясняется уменьшением объема кристаллической фазы.

Снижение интенсивности пика сопровождается образованием „хвоста“ в области более низких волновых чисел. Широкий пик на частоте $480 \mathrm{~cm}^{-1}$, соответствующий аморфной фазе кремния, появляется в спектре образца, облученного дозой $10^{15} \mathrm{~cm}^{-2}$, и растет вместе с дозой облучения.

Так как в спектрах, представленных на рис. 3, наблюдается смещение пика, соответствующего кристаллической фазе при увеличении дозы облучения, можно сделать вывод, что глубина анализа спектроскопии КР в случае пористого кремния не превышает максимальной глубины дефектообразования. В противном случае, спектры содержали бы оба пика: смещенный пик от нанокристаллов и пик от необлученной области на частоте $520 \mathrm{~cm}^{-1}$.

Для оценки среднего диаметра нанокристалла, сравним линию комбинационного рассеяния первого порядка для оптического продольного фононного колебания с экспериментальным спектром.

Линия комбинационного рассеяния первого порядка для оптического продольного фононного колебания может быть представлена как

$$
I_{g}(\omega)=P I_{\mathrm{LO}}(\omega)+Q I_{d}(\omega)+\text { fon, }
$$

где

$$
I_{\mathrm{LO}}(\omega)=\int_{0}^{1} \frac{4 \pi q^{2} \exp \left(-q^{2} L^{2} / 4 a^{2}\right)}{\left[\omega-\omega_{\mathrm{LO}}(\mathbf{q})\right]^{2}+\left[0.5 \Gamma_{c}\right]^{2}} d q
$$

- интенсивность этой линии для нанокристалла сферической формы $[45,46]$, где волновой вектор q выражен в единицах $2 \pi / a(a=0.54 \mathrm{HM}), L-$ диаметр кремниевого нанокристалла, $\omega_{\mathrm{LO}}(\mathbf{q})$ - частота продольных оптических (LO) фононных колебаний вдоль направления [100] в кристалле и $\Gamma_{c}-$ ширина на половине высоты пика кристаллического кремния $\left(5.5 \mathrm{~cm}^{-1}\right)$.

Феноменологическое выражение, следующее из указанного значения дисперсии кривой для LO фонона $\Gamma_{c}$, имеет вид [46]

$$
\omega_{\mathrm{LO}}^{2}(\mathbf{q})=A+B \cos (\pi \mathbf{q} / 2),
$$

где $A=1.7207 \cdot 10^{5} \mathrm{~cm}^{-2}$ и $B=1.0 \cdot 10^{5} \mathrm{~cm}^{-2}$. При $\mathbf{q}=0$ уравнение (2) дает $\omega_{\mathrm{LO}}=521.6 \mathrm{~cm}^{-1}$, что хорошо согласуется со значением частоты LO моды в кристаллическом кремнии.

$$
I_{d}(\omega)=\Gamma_{d} /\left[\left(\omega-\omega_{d}\right)^{2}+\left(0.5 \Gamma_{d}\right)^{2}\right]
$$

- сигнал в спектре КР, соответствующий аморфной фазе [47], $\omega_{d}-$ фононная частота, $\Gamma_{d}-$ полная ширина на половине высоты. fon $=R \omega+S-$ фон. $P, Q, R, S-$ константы.

Моделирование и сравнение линии комбинационного рассеяния первого порядка для оптического продольного фононного колебания с экспериментальным спектром было проведено с помощью пакета MATLAB. Полученные описанным выше способом значения $L$ приведены на рис. 3 и составили 8.0 нм при дозе $10^{15} \mathrm{~cm}^{-2}$ и 5.7 нм при дозе облучения $10^{16} \mathrm{~cm}^{-2}$. С изменением дозы на порядок размер нанокристалла уменьшился в 1.4 раза. При этом распыление материала при указанных условиях эксперимента незначительно. Согласно [48] была оценена толщина кремниевого слоя распыленного ионами гелия при 150 кэВ, дозе облучения $10^{16} \mathrm{~cm}^{-2}$, она составила величину менее монослоя. Таким образом, сдвиг пика комбинационного рассеяния света связан не с физическим уменьшением размера исследуемой области, а с разупорядочением структуры кристаллического остова и образованием нанокристаллов.

\section{4. Заключение}

В представленной работе приведено сравнение процессов дефектообразования в наноразмерном монокристаллическом и пористом кремнии под действием ионного облучения. Пленки монокристаллического кремния были облучены ионами кремния с энергией 200 кэВ и дозами от $4 \cdot 10^{14}$ до $10^{15} \mathrm{~cm}^{-2}$, что соответствует величине смещений на атом от 0.7 до 1.7. Пористый кремний, полученный методом электрохимического травления, был облучен ионами гелия с энергией 150 кэВ, дозами $10^{15}$ и $10^{16} \mathrm{~cm}^{-2}$, что соответствует 0.05 и $0.5 \mathrm{dpa}$. Исследование облученных образцов методом комбинационного рассеяния света показало, что при увеличении дозы облучения пик от кристаллической фазы кремния уменьшается, при этом пик от аморфной фазы растет.

В случае пленки монокристаллического кремния при дозе облучения $10^{15} \mathrm{~cm}^{-2}$ ионами кремния в спектрах 
комбинационного рассеяния света пик от кристаллической фазы практически исчезает, что говорит о разрушении кристаллической решетки. По мере увеличения дозы максимумы пиков от кристаллической и аморфной фаз не смещаются, в то время как для пористого кремния максимум пика, соответствующего кристаллической фазе, смещается в область меньших волновых чисел по мере увеличения дозы облучения.

При близких параметрах смещений на атом 0.7 для монокристаллического и 0.5 для пористого кремния, облученных ионами кремния и гелия соответственно, наблюдается значительно различающаяся картина спектров КР. Для пленки монокристаллического кремния $0.7 \mathrm{dpa} \mathrm{достаточно} \mathrm{для} \mathrm{разрушения} \mathrm{структуры} \mathrm{решетки,} \mathrm{в}$ пористом кремнии наблюдается интенсивный пик, характерный для кристаллической фазы, при значении $0.5 \mathrm{dpa}$, что авторы связывают с наличием нанокристаллов в пористой структуре.

С помощью численного моделирования были определены размеры нанокристаллов в структуре пористого кремния для разных доз облучения. Было показано, что при изменении дозы облучения от $10^{15}$ до $10^{16} \mathrm{~cm}^{-2}$ размер нанокристаллов изменяется от 8 до $5.7 \mathrm{HM}$, что авторы объясняют разрушением структуры кристаллитов под действием ионного облучения.

Исследование выполнено при финансовой поддержке РФФИ в рамках научного проекта № 18-32-01040 мол_а. Авторы выражают благодарность Д.А. Николаеву за проведенное численное моделирование.

\section{Список литературы}

[1] K.A. Gonchar, A.A. Zubairova, A. Schleusener, L.A. Osminkina, V. Sivakov. Nanoscale Res. Lett., 11, 357 (2016).

[2] M.B. Gongalsky, Yu.V. Kargina, L.A. Osminkina, A.M. Perepukhov, M.V. Gulyaev, A.N. Vasiliev, Yu.A. Pirogov, A.V. Maximychev, V.Yu. Timoshenko. Appl. Phys. Lett., 107, 233702 (2015).

[3] R.S. Smerdov, Yu.M. Spivak, V.S. Levitsky, V.A. Moshnikov. IOP Conf. Series: J. Phys.: Conf. Ser., 1038, 012064 (2018).

[4] А.С. Леньшин. ФТП, 52 (3), 342 (2018).

[5] A.A. Shemukhin, Yu.V. Balakshin, V.S. Chernysh, A.S. Patrakeev, S.A. Golubkov, N.N. Egorov, A.I. Sidorov, B.A. Malyukov, V.N. Statsenko, V.D. Chumak. Techn. Phys. Lett., 38 (10), 907 (2012).

[6] V. Parkhutik. Solid-State Electron., 43 (6), 1121 (1999).

[7] Q. Ma, R. Xiong, Y.M. Huang. J. Luminesc., 131 (10), 2053 (2011).

[8] L.T. Canham. Appl. Phys. Lett., 57, 1046 (1990).

[9] M. Saleem, M. Rafiq, S.-Y. Seo, K.H. Lee. Biosci. Rep., 36 (2), 1 (2016).

[10] P.N. Lim, T. Konishia, Z. Wang, J. Feng, L. Wang, J. Han, Z. Yang, E.S. Thian. Mater. Lett., 212, 90 (2018).

[11] W.Y. Tong, M.J. Sweetman, E.R. Marzouk, C. Fraser, T. Kuchel, N.H. Voelcker. Biomaterials, 74, 217 (2016).
[12] E. Tolstik, L.A. Osminkina, C. Matthäus, M. Burkhardt, K.E. Tsurikov, U.A. Natashina, V.Y. Timoshenko, R. Heintzmann, J. Popp, V. Sivakov. Nanomedicine: NBM, 12 (7), 1931 (2016).

[13] Ю.М. Спивак, А.О. Белорус, А.А. Паневин, С.Г. Журавский, В.А. Мошников, К. Беспалова, П.А. Сомов, Ю.М. Жуков, А.С. Комолов, Л.В. Чистякова, Н.Ю. Григорьева. ЖТФ, 88 (9), 1394 (2018).

[14] Song Bai, Ning Zhang, Chao Gao, Yujie Xiong. Nano Energy, 53, 296 (2018).

[15] V.A. Gritsenko, T.V. Perevalov, D.R. Islamov. Phys. Reports, 613, 1 (2016).

[16] V.S. Vendamani, Z.Y. Dang, P. Ramana, A.P. Pathak, V.V. Ravi Kanth Kumar, M.B.H. Breese, S.V.S. Nageswara Rao. Nucl. Instr. Meth. B, 358, 105 (2015).

[17] Yin-Yu Chen, Der-Sheng Chao, Hsu-Sheng Tsai, JenqHorng Liang. Nucl. Instr. Meth. B, 372, 114 (2016).

[18] A.G. Hernández, A.E. Escobosa-Echavarría, Y. Kudriavtsev. Appl. Surf. Sci., 428, 1098 (2018).

[19] V.S. Vendamani, S.V.S. Nageswara Rao, A.P. Pathak. Nucl. Instr. Meth. B, 315, 188 (2013).

[20] S. Hamad, G.K. Podagatlapalli, V.S. Vendamani, S.V.S. Nageswara Rao, A.P. Pathak, S.P. Tewari, S. Venugopal Rao. J. Phys. Chem. C, 118 (13), 7139 (2014).

[21] F. Caridi, A. Picciotto, L. Vanzetti, E. Iacob, C. Scolaro. Radiation Phys. Chem., 115, 49 (2015).

[22] Li-Xin Yang, Yan-Meng Chao, Li Jia, Chao-Bo Li. Appl. Therm. Engin., 99, 253 (2016).

[23] A.E. Ieshkin, S.E. Svyakhovskiy, V.S. Chernysh. Vacuum, 148, 272 (2018).

[24] А.А. Шемухин, Ю.В. Балакшин, В.С. Черныш, С.А. Голубков, Н.Н. Егоров, А.И. Сидоров. ФТП, 48 (4), 535 (2014).

[25] A.A. Shemukhin, A.V. Nazarov, Yu.V. Balakshin, V.S. Chernysh. Nucl. Instr. Meth. B, 354, 274 (2015).

[26] S.P. Low, N.H. Voelcker. In: Handbook of Porous Silicon, ed by L.T. Canham (Springer Int. Publ. Switzerland, 2014) p. 381.

[27] В.А. Юзова, А.А. Левицкий, П.А. Харлашин. Журнал СФУ. Сер.: Техника и технологии, 4 (1), 92 (2011).

[28] Z.C. Feng, R. Tsu. Porous Silicon (Singapore, World Scientific Press, 2014).

[29] К.В. Карабешкин, П.А. Карасёв, А.И. Титов. ФТП, 47 (2), 206 (2013).

[30] R. Herino, G. Bomchil, K. Barla, C. Bertrand, J.L. Ginoux. J. Electrochem. Soc., 134 (8), 1994 (1987).

[31] J.F. Ziegler, M.D. Ziegler, J.P. Biersack. Nucl. Instr. Meth. B, 268, 1818 (2010).

[32] J.F. Ziegler, J.P. Biersack, U. Littmark. The Stopping and Range of Ions in Matter (Pergamon, N.Y., 1985).

[33] A.A. Shemukhin, Yu.V. Balakshin, P.N. Chernykh, V.S. Chernysh. Surface Investigation $X$-ray, Synchrotron and Neutron Techniques, 7, 318 (2013).

[34] A.A. Shemukhin, Yu.V. Balakshin, A.P. Evseev, V.S. Chernysh. Nucl. Instr. Meth. B, 406, 507 (2017).

[35] Q. Nie, Z. Jiang, Z. Gan, S. Liu, H. Yan, H. Fang. J. Cryst. Growth, 488, 1 (2018).

[36] P. Petrik, O. Polgár, T. Lohner, M. Fried, N.Q. Khánh, J. Gyulai. Solid State Phenomena, 82-84, 765 (2002).

[37] К.Д. Кушкина, А.В. Назаров, А.А. Шемухин, А.П. Евсеев. Прикл. физика, 2, 54 (2017).

[38] Yu.V. Balakshin, A.A. Shemukhin, A.V. Nazarov, A.V. Kozhemiako, V.S. Chernysh. Techn. Phys., 63 (12), 1861 (2018). 
[39] R. Prabakaran, R. Kesavamoorthy, S. Amirthapandian, A. Ramanand. Mater. Lett., 58, 3745 (2004).

[40] T.A. Harriman, D.A. Lucca, J.-K. Lee, M.J. Klopfstein, K. Herrmann, M. Nastasi. Nucl. Instr. Meth. B, 267, 1232 (2009).

[41] K.D. Kushkina, A.A. Shemukhin, E.A. Vorobyeva, K.A. Bukunov, A.P. Evseev, A.A. Tatarintsev, K.I. Maslakov, N.G. Chechenin, V.S. Chernysh. Nucl. Instr. Meth. B, 430, 11 (2018).

[42] R. Prabakaran, R. Kesavamoorthy, S. Amirthapandian, Francis P. Xavier. Physica B: Condens. Matter, 337, 36 (3002).

[43] A. Aminzadeh, H. Sarikhani-fard. Spectrochimica Acta, Part A, 55, 1421 (1999).

[44] Д.И. Тетельбаум, А.А. Ежевский, А.Н. Михайлов. ФТП, 37 (11), 1380 (2003).

[45] H. Richter, Z.P. Wang, L. Ley. Solid State Commun., 39, 625 (1981).

[46] I.H. Campbell, P.M. Fauchet. Solid State Commun., 58, 739 (1986).

[47] Dongsheng $\mathrm{Xu}$, Guolin Guo, Linlin Gui, Youqi Tang, B.R. Zhang, G.G. Qin. J. Appl. Phys., 86, 2066 (1999).

[48] Y. Ymamura, H. Tawara. Energy Dependence of Ion-Induced Sputtering Yields from Monoatomic Solids at Normal Incidence (National Institute for Fusion Science, Chigusa-ku, Nagoya 464-01, Japan, 1995).

Редактор Л.В. Шаронова

\section{Features of defect formation in the nanostructured silicon under ion irradiation}

A.V. Kozhemiako ${ }^{1}$, A.P. Evseev ${ }^{1,2}$, Yu.V. Balakshin ${ }^{2}$, A.A. Shemukhin ${ }^{2}$

${ }^{1}$ Lomonosov Moscow State University

(Faculty of Physics),

119991 Moscow, Russia

2 Skobeltsyn Institute of Nuclear Physics,

Lomonosov Moscow State University,

119991 Moscow, Russia

Abstract Irradiations of the nanostructured silicon with $\mathrm{Si}^{+}$and $\mathrm{He}^{+}$ions were carried out with energies of 200 and $150 \mathrm{keV}$, respectively. Raman scattering showed destruction of the structure after irradiations and accumulation of defects at different fluences of irradiation. It is shown that monocrystalline silicon films are amorphized under irradiation at 0.7 displacement per atom. However, porous silicon does not completely amorphize at 0.5 displacement per atom, a weak signal is observed in the Raman spectra corresponding to the amorphous silicon phase, and at the same time there is an obvious signal from the crystalline phase of silicon. The size of nanocrystallites in the structure of porous silicon was estimated at different fluences of irradiation. 